\title{
Fractional Partial Random Differential Equations with State-Dependent Delay
}

\author{
Mouffak Benchohra and Amel Heris
}

\begin{abstract}
In the present paper we provide some existence results for the Darboux problem of partial fractional random differential equations with state-dependent delay by applying the measure of noncompactness and a random fixed point theorem with stochastic domain.
\end{abstract}

AMS Subject Classification (2000). 26A33

Keywords. Random differential equation; Darboux problem; left-sided mixed Riemann-Liouville integral; Caputo fractional order derivative; Measure of noncompactness.

\section{Introduction}

Fractional calculus is generalization of ordinary differentiation and integration to arbitrary non-integer order. The subject is as old as the differential calculus, starting from some speculations of G.W. Leibniz (1967) and L. Euler (1730) and since then, it has continued to be developed up to nowadays. Integral equations are one of the most useful mathematical tools in both pure and applied analysis. This is particulary true of problems in mechanical vibrations and the related fields of engineering and mathematical physics. we can find numerous applications of differential and integral equation of fractional order in finance, hydrology, biophysics, thermodynamics,

The authors are grateful to the referee for the careful reading of the paper. 
control theory, statistical mechanics, astrophysics, cosmology and bioengineering $([14,26,38,39,42])$. There has been a significant development in ordinary and partial fractional differential equations in recent years; see the monographs of Abbas et al. [7,8], Baleanu et al. [14], Kilbas et al. [29], the papers of Abbas et al. [1-6,9,10], Ahmad and Nieto [11], Czlapinski [21,22], Kilbas and Marzan [28], Stanek [41], Vityuk and Golushkov [44], and the references therein.

The theory of functional differential equations has emerged as an important branch of nonlinear analysis. Differential delay equations, or functional differential equations, have been used in modeling scientific phenomena for many years. Often, it has been assumed that the delay is either a fixed constant or is given as an integral in which case it is called a distributed delay; see for instance the books by Hale and Verduyn Lunel [25], Kolmanovskii and Myshkis [30], Smith [40], and Wu [46], and the references therein. However, complicated situations in which the delay depends on the unknown functions have been considered in recent years. These equations are frequently called equations with state-dependent delay, see, for instance $[15-17,20,23,35,36,45]$.

The nature of a dynamic system in engineering or natural sciences depends on the accuracy of the information we have concerning the parameters that describe that system. If the knowledge about a dynamic system is precise then a deterministic dynamical system arises. Unfortunately in most cases the available data for the description and evaluation of parameters of a dynamic system are inaccurate, imprecise or confusing. In other words, evaluation of parameters of a dynamical system is not without uncertainties. When our knowledge about the parameters of a dynamic system are of statistical nature, that is, the information is probabilistic, the common approach in mathematical modeling of such systems is the use of random differential equations or stochastic differential equations. Random differential equations, as natural extensions of deterministic ones, arise in many applications and have been investigated by many mathematicians. We refer the reader to the monographs $[18,31,43]$. The initial value problems for fractional differential with random parameters have been studied by Lupulescu and Ntouyas [33]. The basic tool in the study of the problems for random fractional differential equations is to treat it as a fractional differential equation in some appropriate Banach space. In [34], the authors proved the existence results for a random fractional equation under a Carathéodory condition.

In this paper, we discuss the existence of random solutions for the following fractional partial random differential equations with state-dependent 
delay

$$
\begin{gathered}
\left({ }^{c} D_{0}^{r} u\right)(x, y, w)=f\left(x, y, u_{\left(\rho_{1}\left(x, y, u_{(x, y)}, w\right), \rho_{2}\left(x, y, u_{(x, y)}, w\right)\right)}, w\right), \\
\text { if } J:=[0, a] \times[0, b], w \in \Omega, \\
u(x, y, w)=\phi(x, y, w), \text { if }(x, y) \in \tilde{J}:=[-\alpha, a] \times[-\beta, b] \backslash(0, a] \times(0, b], w \in \Omega, \\
u(x, 0, w)=\varphi(x, w), x \in[0, a], u(0, y, w)=\psi(y, w), y \in[0, b], w \in \Omega,
\end{gathered}
$$

where $\alpha, \beta, a, b>0,{ }^{c} D_{0}^{r}$ is the standard Caputo's fractional derivative of order $r=\left(r_{1}, r_{2}\right) \in(0,1] \times(0,1],(\Omega, \mathcal{A})$ is a measurable space, $f: J \times$ $C \times \Omega \rightarrow E, \rho_{1}, \rho_{2}: J \times C \times \Omega \rightarrow E$ are given functions, $\phi: \tilde{J} \times \Omega \rightarrow E$ is a given continuous function, $\varphi:[0, a] \times \Omega \rightarrow E, \psi:[0, b] \times \Omega \rightarrow E$ are given absolutely continuous functions with $\varphi(x, w)=\phi(x, 0, w), \psi(y, w)=$ $\phi(0, y, w)$ for each $x \in[0, a], y \in[0, b], w \in \Omega,\left(E,\|\cdot\|_{E}\right)$ a separable Banach space, and $C:=C([-\alpha, 0] \times[-\beta, 0], E)$ is the space of continuous functions on $[-\alpha, 0] \times[-\beta, 0]$.

If $u \in C([-\alpha, a] \times[-\beta, b], E) ; a, b, \alpha, \beta>0$ then for any $(x, y) \in J$ define $u_{(x, y)}$ by

$$
u_{(x, y)}(s, t, w)=u(x+s, y+t, w), \text { for }(s, t) \in[-\alpha, 0] \times[-\beta, 0] .
$$

Here $u_{(x, y)}(\cdot, \cdot, w)$ represents the history of the state $u$.

This paper initiates the study of Darboux problem for fractional random partial differential equations with state-dependent delay.

\section{Preliminaries}

In this section, we introduce notations, definitions, and preliminary facts which are used throughout this paper. Denote $L^{1}(J)$ the space of Bochnerintegrable functions $u: J \rightarrow E$ with the norm

$$
\|u\|_{L^{1}}=\int_{0}^{a} \int_{0}^{b}\|u(x, y)\|_{E} d y d x .
$$

$L^{\infty}(J)$ the Banach space of measurable functions $u: J \rightarrow \mathbb{R}$ which are essentially bounded.

As usual, by $A C(J)$ we denote the space of absolutely continuous functions from $J$ into $E$. 
Let $\beta_{E}$ be the $\sigma$-algebra of Borel subsets of $E$. A mapping $v: \Omega \rightarrow E$ is said to be measurable if for any $B \in \beta_{E}$, one has

$$
v^{-1}(B)=\{w \in \Omega: v(w) \in B\} \subset \mathcal{A} .
$$

To define integrals of sample paths of random process, it is necessary to define a jointly measurable map.

Definition 2.1. A mapping $T: \Omega \times E \rightarrow E$ is called jointly measurable if for any $B \in \beta_{E}$, one has

$$
T^{-1}(B)=\{(w, v) \in \Omega \times E: T(w, v) \in B\} \subset \mathcal{A} \times \beta_{E},
$$

where $\mathcal{A} \times \beta_{E}$ is the direct product of the $\sigma$-algebras $\mathcal{A}$ and $\beta_{E}$ those defined in $\Omega$ and $E$ respectively.

Lemma 2.1. Let $T: \Omega \times E \rightarrow E$ be a mapping such that $T(\cdot, v)$ is measurable for all $v \in E$, and $T(w, \cdot)$ is continuous for all $w \in \Omega$. Then the map $(w, v) \mapsto$ $T(w, v)$ is jointly measurable.

Definition 2.2. A function $f: J \times E \times \Omega \rightarrow E$ is called random Carathéodory if the following conditions are satisfied:

(i) The map $(x, y, w) \rightarrow f(x, y, u, w)$ is jointly measurable for all $u \in E$, and

(ii) The map $u \rightarrow f(x, y, u, w)$ is continuous for almost all $(x, y) \in J$ and $w \in \Omega$.

Let $T: \Omega \times E \rightarrow E$ be a mapping. Then $T$ is called a random operator if $T(w, u)$ is measurable in $w$ for all $u \in E$ and it is expressed as $T(w) u=T(w, u)$. In this case we also say that $T(w)$ is a random operator on $E$. A random operator $T(w)$ on $E$ is called continuous (resp. compact, totally bounded and completely continuous) if $T(w, u)$ is continuous (resp. compact, totally bounded and completely continuous) in $u$ for all $w \in \Omega$. The details of completely continuous random operators in Banach spaces and their properties appear in Itoh [27].

Definition 2.3. [24] Let $\mathcal{P}(Y)$ be the family of all nonempty subsets of $Y$ and $C$ be a mapping from $\Omega$ into $\mathcal{P}(Y)$. A mapping $T:\{(w, y): w \in \Omega, y \in$ $C(w)\} \rightarrow Y$ is called random operator with stochastic domain $C$ if $C$ is measurable (i.e., for all closed $A \subset Y,\{w \in \Omega, C(w) \cap A \neq \emptyset\}$ is measurable) and for all open $D \subset Y$ and all $y \in Y,\{w \in \Omega: y \in C(w), T(w, y) \in D\}$ is 
measurable. $T$ will be called continuous if every $T(w)$ is continuous. For a random operator $T$, a mapping $y: \Omega \rightarrow Y$ is called random (stochastic) fixed point of $T$ if for $P$-almost all $w \in \Omega, y(w) \in C(w)$ and $T(w) y(w)=y(w)$ and for all open $D \subset Y,\{w \in \Omega: y(w) \in D\}$ is measurable.

Let $\mathcal{M}_{X}$ denote the class of all bounded subsets of a metric space $X$.

Definition 2.4. Let $X$ be a complete metric space. A map $\alpha: \mathcal{M}_{X} \rightarrow$ $[0, \infty)$ is called a measure of noncompactness on $X$ if it satisfies the following properties for all $B, B_{1}, B_{2} \in \mathcal{M}_{X}$.

(MNC.1) $\alpha(B)=0$ if and only if $B$ is precompact (Regularity),

(MNC.2) $\alpha(B)=\alpha(\bar{B})$ (Invariance under closure),

$(M N C .3) \alpha\left(B_{1} \cup B_{2}\right)=\max \left\{\alpha\left(B_{1}\right), \alpha\left(B_{2}\right)\right\}$ (Semi-additivity).

For more details on measure of noncompactness and its properties see $[12,13]$.

Example 2.1. In every metric space $X$, the map $\phi: \mathcal{M}_{X} \rightarrow[0, \infty)$ with $\phi(B)=0$ if $B$ is relatively compact and $\phi(B)=1$ otherwise is a measure of noncompactness, the so-called discrete measure of noncompactness [[13], Example1, p. 19].

Let $\theta=(0,0), r_{1}, r_{2}>0$ and $r=\left(r_{1}, r_{2}\right)$. For $f \in L^{1}(J)$, the expression

$$
\left(I_{\theta}^{r} f\right)(x, y)=\frac{1}{\Gamma\left(r_{1}\right) \Gamma\left(r_{2}\right)} \int_{0}^{x} \int_{0}^{y}(x-s)^{r_{1}-1}(y-t)^{r_{2}-1} f(s, t) d t d s,
$$

is called the left-sided mixed Riemann-Liouville integral of order $r$, where $\Gamma(\cdot)$ is the (Euler's) Gamma function defined by

$$
\Gamma(\xi)=\int_{0}^{\infty} t^{\xi-1} e^{-t} d t ; \xi>0 .
$$

In particular,

$\left(I_{\theta}^{\theta} u\right)(x, y)=u(x, y),\left(I_{\theta}^{\sigma} u\right)(x, y)=\int_{0}^{x} \int_{0}^{y} u(s, t) d t d s ;$ for almost all $(x, y) \in J$

where $\sigma=(1,1)$.

For instance, $I_{\theta}^{r} u$ exists for all $r_{1}, r_{2} \in(0, \infty)$, when $u \in L^{1}(J)$. Note also that when $u \in \mathcal{C}$, then $\left(I_{\theta}^{r} u\right) \in \mathcal{C}$, moreover

$$
\left(I_{\theta}^{r} u\right)(x, 0)=\left(I_{\theta}^{r} u\right)(0, y)=0 ; x \in[0, a], y \in[0, b] .
$$


By $1-r$ we mean $\left(1-r_{1}, 1-r_{2}\right) \in[0,1) \times[0,1)$. Denote by $D_{x y}^{2}:=\frac{\partial^{2}}{\partial x \partial y}$, the mixed second order partial derivative.

Definition 2.5. [44] Let $r \in(0,1] \times(0,1]$ and $u \in L^{1}(J)$. The Caputo fractional-order derivative of order $r$ of $u$ is defined by the expression

$$
{ }^{c} D_{\theta}^{r} u(x, y)=\left(I_{\theta}^{1-r} D_{x y}^{2} u\right)(x, y) .
$$

The case $\sigma=(1,1)$ is included and we have

$$
\left({ }^{c} D_{\theta}^{\sigma} u\right)(x, y)=\left(D_{x y}^{2} u\right)(x, y) ; \text { for almost all }(x, y) \in J .
$$

Lemma 2.2. [19] If $Y$ is a bounded subset of Banach space $X$, then for each $\epsilon>0$, there is a sequence $\left\{y_{k}\right\}_{k=1}^{\infty} \subset Y$ such that

$$
\alpha(Y) \leq 2 \alpha\left(\left\{y_{k}\right\}_{k=1}^{\infty}\right)+\epsilon
$$

Lemma 2.3. [37] If $\left\{u_{k}\right\}_{k=1}^{\infty} \subset L^{1}(J)$ is uniformly integrable, then $\alpha\left(\left\{u_{k}\right\}_{k=1}^{\infty}\right)$ is measurable and for each $(x, y) \in J$,

$$
\alpha\left(\left\{\int_{0}^{x} \int_{0}^{y} u_{k}(s, t) d t d s\right\}_{k=1}^{\infty}\right) \leq 2 \int_{0}^{x} \int_{0}^{y} \alpha\left(\left\{u_{k}(s, t)\right\}_{k=1}^{\infty}\right) d t d s .
$$

Lemma 2.4. [32] Let $F$ be a closed and convex subset of a real Banach space, let $G: F \rightarrow F$ be a continuous operator and $G(F)$ be bounded. If there exists a constant $k \in[0,1)$ such that for each bounded subset $B \subset F$,

$$
\alpha(G(B)) \leq k \alpha(B)
$$

then $G$ has a fixed point in $F$.

\section{Existence Results}

We need the following lemma:

Lemma 3.1. $[2,7]$ Let $h \in L^{1}(J)$. Then the linear problem

$$
\left\{\begin{array}{l}
{ }^{c} D_{\theta}^{r} u(x, y)=h(x, y) ; \text { for a.a. }(x, y) \in J:=[0, a] \times[0, b] \\
u(x, 0)=\varphi(x) ; x \in[0, a] \\
u(0, y)=\psi(y) ; y \in[0, b] \\
\varphi(0)=\psi(0) .
\end{array}\right.
$$


has a unique solution u given by

$$
u(x, y)=\mu(x, y)+I_{\theta}^{r} h(x, y) ; \quad \text { for a.a. }(x, y) \in J,
$$

where

$$
\mu(x, y)=\varphi(x)+\psi(y)-\varphi(0)
$$

Let us assume that the function $f$ is random Carathéodory on $J \times C \times \Omega$. From Lemma 3.1, we have the following lemma.

Lemma 3.2. Let $0<r_{1}, r_{2} \leq 1, \mu(x, y, w)=\varphi(x, w)+\psi(y, w)-\varphi(0, w)$. A function $u \in \Omega \times C_{(a, b)}$ is a solution of the random problem (1.1)-(1.3) if $u$ satisfies condition (1.2) for $(x, y) \in \tilde{J}, w \in \Omega$ and $u$ is a solution of the equation

$$
\begin{gathered}
u(x, y, w)= \\
\mu(x, y, w)+\int_{0}^{x} \int_{0}^{y} \frac{(x-s)^{r_{1}-1}(y-t)^{r_{2}-1}}{\Gamma\left(r_{1}\right) \Gamma\left(r_{2}\right)} f\left(s, t, u_{\left(\rho_{1}\left(s, t, u_{(s, t)}\right), \rho_{2}\left(s, t, u_{(s, t)}\right)\right)}, w\right) d t d s \\
\text { for }(x, y) \in J, w \in \Omega
\end{gathered}
$$

Set $\mathcal{R}:=\mathcal{R}_{\left(\rho_{1}^{-}, \rho_{2}^{-}\right)}$

$$
\begin{gathered}
=\left\{\left(\rho_{1}(s, t, u, w), \rho_{2}(s, t, u, w)\right):(s, t) \in J, u_{(s, t)}(\cdot, \cdot, w) \in C, w \in \Omega,\right. \\
\left.\rho_{i}(s, t, u, w) \leq 0 ; i=1,2\right\} .
\end{gathered}
$$

We always assume that $\rho_{i}: J \times C \times \Omega \rightarrow E ; i=1,2$ are continuous and the function $(s, t) \longmapsto u_{(s, t)}$ is continuous from $\mathcal{R}$ into $C$.

The following hypotheses will be used in the sequel.

$\left(H_{1}\right)$ The functions $w \mapsto \varphi(x, 0, w)$ and $w \mapsto \psi(0, y, w)$ are measurable and bounded for a.e. $x \in[0, a]$ and $y \in[0, b]$ respectively,

$\left(H_{2}\right)$ The function $\Phi$ is measurable for $(x, y) \in \tilde{J}$

$\left(H_{3}\right)$ The function $f$ is random Carathéodory on $J \times C \times \Omega$,

$\left(H_{4}\right)$ There exist functions $p_{1}, p_{2}: J \times \Omega \rightarrow[0, \infty)$ with $p_{i}(\cdot, w) \in L^{\infty}(J,[0, \infty)) ; i=$ 1,2 such that for each $w \in \Omega$,

$$
\|f(x, y, u, w)\|_{E} \leq p_{1}(x, y, w)+p_{2}(x, y, w)\|u\|_{C},
$$

for all $u \in C$ and a.e. $(x, y) \in J$, 
$\left(H_{5}\right)$ For any bounded $B \subset E$,

$$
\alpha(f(x, y, B, w)) \leq p_{2}(x, y, w) \alpha(B), \text { for a.e. }(x, y) \in J,
$$

$\left(H_{6}\right)$ There exists a random function $R: \Omega \rightarrow(0, \infty)$ such that

$$
\mu^{*}(w)+\frac{\left(p_{1}^{*}(w)+p_{2}^{*}(w) R(w)\right) a^{r_{1}} b^{r_{2}}}{\Gamma\left(1+r_{1}\right) \Gamma\left(1+r_{2}\right)} \leq R(w),
$$

where

$$
\mu^{*}(w)=\sup _{(x, y) \in J}\|\mu(x, y, w)\|_{E}, p_{i}^{*}(w)=\sup \operatorname{ess}_{(x, y) \in J} p_{i}(x, y, w) ; i=1,2,
$$

Theorem 3.3. Assume that hypotheses $\left(H_{1}\right)-\left(H_{6}\right)$ hold. If

$$
\ell:=\frac{4 p_{2}^{*}(w) a^{r_{1}} b^{r_{2}}}{\Gamma\left(1+r_{1}\right) \Gamma\left(1+r_{2}\right)}<1
$$

then the problem (1.1)-(1.3) has a random solution defined on $[-\alpha, a] \times$ $[-\beta, b]$.

Proof. Define the operator $N: \Omega \times C_{(a, b)} \rightarrow C_{(a, b)}$ by

$$
(N(w) u)(x, y)=\left\{\begin{array}{l}
\phi(x, y, w), \quad(x, y) \in \tilde{J}, w \in \Omega \\
\mu(x, y, w)+\frac{1}{\Gamma\left(r_{1}\right) \Gamma\left(r_{2}\right)} \int_{0}^{x} \int_{0}^{y}(x-s)^{r_{1}-1}(y-t)^{r_{2}-1} \\
\times f\left(s, t, u_{\left.\left(\rho_{1}\left(s, t, u_{(s, t)}, w\right), \rho_{2}\left(s, t, u_{(s, t)}, w\right)\right), w\right)} d t d s\right. \\
\quad(x, y) \in J, w \in \Omega .
\end{array}\right.
$$

Since the functions $\varphi, \psi$ and $f$ are absolutely continuous, the function $\mu$ and the indefinite integral are absolutely continuous for all $w \in \Omega$ and almost all $(x, y) \in J$. Again, as the map $\mu$ is continuous for all $w \in \Omega$ and the indefinite integral is continuous on $J$, then $N(w)$ defines a mapping $N$ : $\Omega \times C_{(a, b)} \rightarrow C_{(a, b)}$. Hence $u$ is a solution for the problem (1.1)-(1.3) if and only if $u=(N(w)) u$. We shall show that the operator $N$ satisfies all conditions of Lemma 2.4. The proof will be given in several steps.

Step 1: $N(w)$ is a random operator with stochastic domain on $C_{(a, b)}$. Since $f(x, y, u, w)$ is random Carathéodory, the map $w \rightarrow f(x, y, u, w)$ is measurable in view of Definition 2.1. Similarly, the function $(s, t) \mapsto(x-$ $s)^{r_{1}-1}(y-t)^{r_{2}-1} f\left(s, t, u_{(s, t)}, w\right)$ is measure as the product of a continuous and a measurable function. Further, the integral is a limit of a finite sum of measurable functions, therefore, the map

$$
w \mapsto \mu(x, y, w)+
$$


$\frac{1}{\Gamma\left(r_{1}\right) \Gamma\left(r_{2}\right)} \int_{0}^{x} \int_{0}^{y}(x-s)^{r_{1}-1}(y-t)^{r_{2}-1} f\left(s, t, u_{\left(\rho_{1}\left(s, t, u_{(s, t)}\right), \rho_{2}\left(s, t, u_{(s, t)}\right)\right)}, w\right) d t d s$ is measurable. As a result, $N$ is a random operator on $\Omega \times C_{(a, b)}$ into $C_{(a, b)}$.

Let $W: \Omega \rightarrow \mathcal{P}\left(C_{(a, b)}\right)$ be defined by

$$
W(w)=\left\{u \in C_{(a, b)}:\|u\|_{\infty} \leq R(w)\right\},
$$

with $R(\cdot)$ is chosen appropriately. From instance, we assume that

$$
R(w) \geq \frac{\mu^{*}+p_{1}^{*}(w) \frac{a^{r_{1}} b^{r_{2}}}{\Gamma\left(1+r_{1}\right) \Gamma\left(1+r_{2}\right)}}{1-p_{2}^{*}(w) \frac{a^{r_{1} b^{r}}}{\Gamma\left(1+r_{1}\right) \Gamma\left(1+r_{2}\right)}} .
$$

Clearly, $W(w)$ is bounded, closed, convex and solid for all $w \in \Omega$. Then $W$ is measurable by Lemma 17 of [24]. Let $w \in \Omega$ be fixed, then from $\left(H_{4}\right)$, for any $u \in W(w)$, we get

$$
\begin{aligned}
& \|(N(w) u)(x, y)\|_{E} \\
& \leq\|\mu(x, y, w)\|_{E}+ \\
& \int_{0}^{x} \int_{0}^{y} \frac{(x-s)^{r_{1}-1}(y-t)^{r_{2}-1}}{\Gamma\left(r_{1}\right) \Gamma\left(r_{2}\right)}\left\|f\left(s, t, u_{\left(\rho_{1}\left(s, t, u_{(s, t)}\right), \rho_{2}\left(s, t, u_{(s, t)}\right)\right)}, w\right)\right\|_{E} d t d s \\
& \leq\|\mu(x, y, w)\|_{E}+\frac{1}{\Gamma\left(r_{1}\right) \Gamma\left(r_{2}\right)} \int_{0}^{x} \int_{0}^{y}(x-s)^{r_{1}-1}(y-t)^{r_{2}-1} p_{1}(s, t, w) d t d s \\
& \quad+\frac{1}{\Gamma\left(r_{1}\right) \Gamma\left(r_{2}\right)} \int_{0}^{x} \int_{0}^{y}(x-s)^{r_{1}-1}(y-t)^{r_{2}-1} p_{2}(s, t, w)\left\|u_{\left(\rho_{1}\left(s, t, u_{(s, t)}\right), \rho_{2}\left(s, t, u_{(s, t)}\right)\right)}\right\|_{E} d t d s \\
& \leq \mu^{*}(w)+\frac{p_{1}^{*}(w)}{\Gamma\left(r_{1}\right) \Gamma\left(r_{2}\right)} \int_{0}^{x} \int_{0}^{y}(x-s)^{r_{1}-1}(y-t)^{r_{2}-1} d t d s \\
& \quad+\frac{p_{2}^{*}(w) R(w)}{\Gamma\left(r_{1}\right) \Gamma\left(r_{2}\right)} \int_{0}^{x} \int_{0}^{y}(x-s)^{r_{1}-1}(y-t)^{r_{2}-1} d t d s \\
& \leq \mu^{*}(w)+\frac{\left(p_{1}^{*}(w)+p_{2}^{*}(w) R(w)\right) a^{r_{1}} b^{r_{2}}}{\Gamma\left(1+r_{1}\right) \Gamma\left(1+r_{2}\right)} \\
& \leq R(w) .
\end{aligned}
$$

Therefore, $N$ is a random operator with stochastic domain $W$ and $N(w)$ : $W(w) \rightarrow N(w)$. Furthermore, $N(w)$ maps bounded sets into bounded sets in $C_{(a, b)}$.

Step 2: $N(w)$ is continuous.

Let $\left\{u_{n}\right\}$ be a sequence such that $u_{n} \rightarrow u$ in $C_{(a, b)}$. Then, for each $(x, y) \in J$ and $w \in \Omega$, we have

$$
\begin{aligned}
& \left\|\left(N(w) u_{n}\right)(x, y)-(N(w) u)(x, y)\right\|_{E} \leq \frac{1}{\Gamma\left(r_{1}\right) \Gamma\left(r_{2}\right)} \int_{0}^{x} \int_{0}^{y}(x-s)^{r_{1}-1}(y-t)^{r_{2}-1} \\
& \times\left\|f\left(s, t, u_{n\left(\rho_{1}\left(s, t, u_{(s, t)}\right), \rho_{2}\left(s, t, u_{(s, t)}\right)\right)}, w\right)-f\left(s, t, u_{\left(\rho_{1}\left(s, t, u_{(s, t)}\right), \rho_{2}\left(s, t, u_{(s, t)}\right)\right)}, w\right)\right\|_{E} d t d s .
\end{aligned}
$$


Using the Lebesgue dominated convergence theorem, we get

$$
\left\|N(w) u_{n}-N(w) u\right\|_{\infty} \rightarrow 0 \text { as } n \rightarrow \infty .
$$

As a consequence of Steps 1 and 2, we can conclude that $N(w): W(w) \rightarrow$ $N(w)$ is a continuous random operator with stochastic domain $W$, and $N(w)(W(w))$ is bounded.

Step 3: For each bounded subset $B$ of $W(w)$ we have

$$
\alpha(N(w) B) \leq \ell \alpha(B) .
$$

Let $w \in \Omega$ be fixed. From Lemmas 2.2 and 2.3, for any $B \subset W$ and any $\epsilon>0$, there exists a sequence $\left\{u_{n}\right\}_{n=0}^{\infty} \subset B$, such that for all $(x, y) \in J$, we have

$$
\begin{aligned}
& \alpha((N(w) B)(x, y)) \\
& =\alpha(\{\mu(x, y)+ \\
& \left.\left.\int_{0}^{x} \int_{0}^{y} \frac{(x-s)^{r_{1}-1}(y-t)^{r_{2}-1}}{\Gamma\left(r_{1}\right) \Gamma\left(r_{2}\right)} f\left(s, t, u_{\left(\rho_{1}\left(s, t, u_{(s, t)}\right), \rho_{2}\left(s, t, u_{(s, t)}\right)\right)}, w\right) d t d s ; u \in B\right\}\right) \\
& \leq 2 \alpha\left(\left\{\int_{0}^{x} \int_{0}^{y} \frac{(x-s)^{r_{1}-1}(y-t)^{r_{2}-1}}{\Gamma\left(r_{1}\right) \Gamma\left(r_{2}\right)} f\left(s, t, u_{n\left(\rho_{1}\left(s, t, u_{(s, t)}\right), \rho_{2}\left(s, t, u_{(s, t)}\right)\right)}, w\right) d t d s\right\}_{n=1}^{\infty}\right)+\epsilon \\
& \leq 4 \int_{0}^{x} \int_{0}^{y} \alpha\left(\left\{\frac{(x-s)^{r_{1}-1}(y-t)^{r_{2}-1}}{\Gamma\left(r_{1}\right) \Gamma\left(r_{2}\right)} f\left(s, t, u_{n\left(\rho_{1}\left(s, t, u_{(s, t)}\right), \rho_{2}\left(s, t, u_{(s, t)}\right)\right)}, w\right)\right\}_{n=1}^{\infty}\right) d t d s+\epsilon \\
& \leq 4 \int_{0}^{x} \int_{0}^{y} \frac{(x-s)^{r_{1}-1}(y-t)^{r_{2}-1}}{\Gamma\left(r_{1}\right) \Gamma\left(r_{2}\right)} \alpha\left(\left\{f\left(s, t, u_{n\left(\rho_{1}\left(s, t, u_{(s, t)}\right), \rho_{2}\left(s, t, u_{(s, t)}\right)\right)}, w\right)\right\}_{n=1}^{\infty}\right) d t d s+\epsilon \\
& \leq 4 \int_{0}^{x} \int_{0}^{y} \frac{(x-s)^{r_{1}-1}(y-t)^{r_{2}-1}}{\Gamma\left(r_{1}\right) \Gamma\left(r_{2}\right)} p_{2}(s, t, w) \alpha\left(\left\{u_{n\left(\rho_{1}\left(s, t, u_{(s, t)}\right), \rho_{2}\left(s, t, u_{(s, t)}\right)\right)}\right\}_{n=1}^{\infty}\right) d t d s+\epsilon \\
& \leq\left(4 \int_{0}^{x} \int_{0}^{y} \frac{(x-s)^{r_{1}-1}(y-t)^{r_{2}-1}}{\Gamma\left(r_{1}\right) \Gamma\left(r_{2}\right)} p_{2}(s, t, w) d s d t\right) \alpha\left(\left\{u_{n}\right\}_{n=1}^{\infty}\right)+\epsilon \\
& \leq\left(4 \int_{0}^{x} \int_{0}^{y} \frac{(x-s)^{r_{1}-1}(y-t)^{r_{2}-1}}{\Gamma\left(r_{1}\right) \Gamma\left(r_{2}\right)} p_{2}(s, t, w) d t d s\right) \alpha(B)+\epsilon \\
& \leq \frac{4 p_{2}^{*}(w) a^{r_{1}} b^{r_{2}}}{\Gamma\left(1+r_{1}\right) \Gamma\left(1+r_{2}\right)} \alpha(B)+\epsilon \\
& =\ell \alpha(B)+\epsilon
\end{aligned}
$$

Since $\epsilon>0$ is arbitrary, then

$$
\alpha(N(B)) \leq \ell \alpha(B) .
$$

It follows from Lemma 2.4 that for each $w \in \Omega, N$ has at least one fixed point in $W$. Since $\bigcap_{w \in \Omega} \operatorname{int} W(w) \neq \emptyset$, and a measurable selector of int $W$ exists, 
Lemma 2.4 implies that $N$ has a stochastic fixed point, i.e., the problem (1.1)-(1.3) has at least one random solution on $C_{(a, b)}$.

\section{An Example}

Let $E=\mathbb{R}, \Omega=(-\infty, 0)$ be equipped with the usual $\sigma$-algebra consisting of Lebesgue measurable subsets of $(-\infty, 0)$. Consider the following partial functional random differential equation of the form

$$
\begin{gathered}
\left({ }^{c} D_{0}^{r} u\right)(x, y)=\frac{\left|u\left(x-\sigma_{1}(u(x, y, w)), y-\sigma_{2}(u(x, y, w)), w\right)\right|+2}{e^{x+y+4}\left(1+w^{2}+\left|u\left(x-\sigma_{1}(u(x, y, w)), y-\sigma_{2}(u(x, y, w)), w\right)\right|\right)} \\
\quad \text { if }(x, y) \in[0,1] \times[0,1], \quad(4.1) \\
u(x, y, w)=x \sin w+y^{2} \cos w,(x, y) \in[-1,1] \times[-2,1] \backslash(0,1] \times(0,1], w \in \Omega, \\
u(x, 0, w)=x \sin w ; x \in[0,1], u(0, y, w)=y^{2} \cos w ; y \in[0,1], w \in \Omega,
\end{gathered}
$$

where $\sigma_{1} \in C(\mathbb{R},[0,1]), \sigma_{2} \in C(\mathbb{R},[0,2])$. Set

$$
\begin{aligned}
& \rho_{1}(x, y, \varphi, w)=x-\sigma_{1}(\varphi(0,0, w)), \\
& \rho_{2}(x, y, \varphi, w)=y-\sigma_{2}(\varphi(0,0, w)),
\end{aligned}
$$

where $(x, y) \in J, \varphi(\cdot, \cdot, w) \in C([-1,0] \times[-2,0], \mathbb{R}), w \in \Omega$,

$$
\begin{gathered}
f(x, y, u, w)=\frac{|u|+2}{\left(e^{x+y+4}\right)\left(1+w^{2}+|u|\right)}, \quad(x, y) \in[0,1] \times[0,1], \\
u \in C([-1,0] \times[-2,0], \mathbb{R}) .
\end{gathered}
$$

The functions

$$
\begin{gathered}
w \mapsto \varphi(x, 0, w)=x \sin w, w \mapsto \psi(0, y, w)=y^{2} \cos w \\
w \mapsto \Phi(x, y, w)=x \sin w+y^{2} \cos w
\end{gathered}
$$

are measurable and bounded with

$$
|\varphi(x, 0, w)| \leq 1,|\psi(0, y, w)| \leq 1
$$

hence, condition $\left(H_{1}\right)$ is satisfied.

Clearly, the map $(x, y, w) \mapsto f(x, y, u, w)$ is jointly continuous for all $u \in \mathbb{R}$ and hence jointly measurable for all $u \in \mathbb{R}$. Also the map $u \mapsto f(x, y, u, w)$ is 
continuous for all $(x, y) \in J$ and $w \in \Omega$. So the function $f$ is Carathéodory on $[0,1] \times[0,1] \times \mathbb{R} \times \Omega$.

For each $\varphi \in C([-1,0] \times[-2,0], \mathbb{R}),(x, y) \in[0,1] \times[0,1]$ and $w \in \Omega$, we have

$$
|f(x, y, u, w)| \leq 1+\frac{1}{e^{4}}|u| .
$$

Hence condition $\left(H_{4}\right)$ is satisfied with

$$
p_{1}(x, y, w)=p_{1}^{*}=1, \quad p_{2}(x, y, w)=p_{2}^{*}=\frac{1}{e^{4}}
$$

Also, the conditions $\left(H_{5}\right)$ is satisfied.

We shall show that condition $\ell<1$ holds with $a=b=1$. Indeed, for each $\left(r_{1}, r_{2}\right) \in(0,1] \times(0,1]$ we have

$$
\begin{aligned}
\ell & =\frac{4 p_{2}^{*} a^{r_{1}} b^{r_{2}}}{\Gamma\left(1+r_{1}\right) \Gamma\left(1+r_{2}\right)} \\
& =\frac{4}{e^{4} \Gamma\left(1+r_{1}\right) \Gamma\left(1+r_{2}\right)} \\
& <1 .
\end{aligned}
$$

Consequently, Theorem 3.3 implies that the problem $(4.1)-(4.3)$ has a random solution defined on $[-1,1] \times[-2,1]$.

\section{References}

[1] S. Abbas D. Baleanu and M. Benchohra, Global attractivity for fractional order delay partial integro-differential equations, Adv. Difference Equ, 2012, (2012), 19.

[2] S. Abbas and M. Benchohra, Darboux problem for perturbed partial differential equations of fractional order with finite delay, Nonlinear Anal. Hybrid Syst, 3, (2009), 597-604.

[3] S. Abbas and M. Benchohra, Fractional order partial hyperbolic differential equations involving Caputo's derivative, Stud. Univ. Babeş-Bolyai Math, 57, (2012), 469 479.

[4] S. Abbas and M. Benchohra, Upper and lower solutions method for Darboux problem for fractional order implicit impulsive partial hyperbolic differential equations, Acta Univ. Palacki. Olomuc, 51, (2012), 5-18.

[5] S. Abbas M. Benchohra and A. Cabada, Partial neutral functional integrodifferential equations of fractional order with delay, Bound. Value Prob, 2012, (2012), 128. 
[6] S. Abbas M. Benchohra and L. Gorniewicz, Existence theory for impulsive partial hyperbolic functional differential equations involving the Caputo fractional derivative, Sci. Math. Jpn, (2010), 271-282.

[7] S. Abbas M. Benchohra and G.M. N'Guérékata, Topics in Fractional Differential Equations, Springer, New York, 2012.

[8] S. Abbas M. Benchohra and G.M. N'Guérékata, Advanced Fractional Differential and Integral Equations, Nova Science Publishers, New York, 2015.

[9] S. Abbas M. Benchohra and A.N.Vityuk, On fractional order derivatives and Darboux problem for implicit differential equations, Frac. Calc. Appl. Anal, 15, (2012), 168-182.

[10] S. Abbas M. Benchohra and Y. Zhou, Darboux problem for tractional order neutral functional partial hyperbolic differential equations, Int. J. Dynam. Systems Differ. Equa, 2, (2009), 301-312.

[11] B. Ahmad and J.J. Nieto, Riemann-Liouville fractional differential equations with fractional boundary conditions, Social Text, 13, (2012), 329-336.

[12] J. Appell, Implicit functions, nonlinear integral equations, and the measure of noncompactness of the superposition operator, J. Math. Anal. Appl, 83, (1981), 251-263.

[13] J. M. Ayerbee Toledano T. Dominguez Benavides and G. Lopez Acedo, Measures of noncompactness in metric fixed point theory, Operator Theory, Advances and Applications, Birkhäuser, Berlin, 1997.

[14] D. Baleanu K. Diethelm E. Scalas and J.J. Trujillo, Fractional Calculus Models and Numerical Methods, World Scientific Publishing, New York, 2012.

[15] J. Belair, Population models with state-dependent delays, Lect. Notes Pure Appl. Maths., Dekker, New York, 131, (1990), 156-176.

[16] J. Bélair and M.C. Mackey, Consumer memory and price fluctuations on commodity markets: An intergrodifferential model, J. Dynam. Differential Equations, 1, (1989), 299-325.

[17] J. Belair M. C. Mackey and J. Mahaffy, Age-structured and two delay models for erythropoiesis, Math. Biosciences, 128, (1995), 317-346.

[18] A.T. Bharucha-Reid, Random Integral Equations, Academic Press, New York

[19] D. Bothe, Multivalued perturbation of m-accretive differential inclusions, Isr. J. Math, 108, (1998), 109-138.

[20] F. Chen D. Sun and J. Shi, Periodicity in a food-limited population model with toxicants and state-dependent delays, J. Math. Anal. Appl, 288, (2003), 136-146.

[21] T. Czlapinski, On the Darboux problem for partial differential-functional equations with infinite delay at derivatives, Nonlinear Anal, 44, (2001), 389-398.

[22] T. Czlapinski, Existence of solutions of the Darboux problem for partial differentialfunctional equations with infinite delay in a Banach space, Comment. Math. Prace Mat., 35, (1995), 111-122.

[23] R.D. Driver and M.J. Norrisn, Note on uniqueness for a one-dimentional twobody problem of classical electrodynamics, Ann. Phys, 42, (1964), 347-351. 
[24] H. W. Engl, A general stochastic fixed-point theorem for continuous random operators on stochastic domains, J. Math. Anal. Appl, 66, (1978), 220-231.

[25] J. K. Hale and S. Verduyn Lunel, Introduction to Functional-Differential Equations, Springer, New York, 1993.

[26] R. Hilfer, Applications of Fractional Calculus in Physics, World Scientific, Singapore, 2000.

[27] S. Itoh, Random fixed point theorems with applications to random differential equations in Banach spaces, J. Math. Anal. Appl, 67, (1979), 261-273.

[28] A. A. Kilbas and S. A. Marzan, Nonlinear differential equations with the Caputo fractional derivative in the space of continuously differentiable functions, Differential Equations, 41, (2005), 84-89.

[29] A. A. Kilbas H. M. Srivastava and J. J. Trujillo, Theory and Applications of Fractional Differential Equations, Elsevier Science B.V, Amsterdam, 2006.

[30] V. Kolmanovskii and A. Myshkis, Introduction to the Theory and Applications of Functional-Differential Equations, Kluwer Academic Publishers, Dordrecht, 1999.

[31] G.S. Ladde and V. Lakshmikantham, Random Differential Inequalities, Academic Press, New York, 1980.

[32] L. Liu F. Guo C. Wu and Y. Wu, Existence theorems of global solutions for nonlinear Volterra type integral equations in Banach spaces, J. Math. Anal. Appl, 309, (2005), 638-649.

[33] V. Lupulescu and S.K. Ntouyas, Random fractional differential equations, Int. Electron. J. Pure Appl. Math, 4, (2012), 119-136.

[34] V. Lupulescu D. O'Regan and Ghaus ur Rahman, Existence results for random fractional differential equations, Opuscula Math, 34, (2014), 813-825.

[35] M.C. Mackey, Commodity price fluctuations: price dependent delays and nonlinearities as explanatory factors, J. Econ. Theory, 48, (1989), 479-59.

[36] M.C. Mackey and J. Milton, Feedback delays and the orign of blood cell dynamics, Comm. Theor. Biol, 1, (1990), 299-372.

[37] H. Mönch, Boundary value problems for nonlinear ordinary differential equations of second order in Banach spaces, Nonlinear Anal., Theory Methods Appl, 4, (1980), 985-999.

[38] M. D. Ortigueira, Fractional Calculus for Scientists and Engineers, Springer, Dordrecht, 2011.

[39] I. Podlubny, Fractional Differential Equations, Academic Press, San Diego, 1999.

[40] H. Smith, An Introduction to Delay Differential Equations with Applications to the Life Sciences, Springer, 2011.

[41] S. Stanek, Limit properties of positive solutions of fractional boundary value problems, Appl. Math. Comput, 219, (2012), 2361-2370.

[42] V. E. Tarasov, Fractional Dynamics. Applications of Fractional Calculus to Dynamics of Particles, Fields and Media, Springer, Heidelberg, 2010. 
[43] C.P. Tsokos and W.J. Padgett, Random Integral Equations with Applications to Life Sciences and Engineering, Academic Press, New York, 1974.

[44] A. N. Vityuk and A. V. Golushkov, Existence of solutions of systems of partial differential equations of fractional order, Nonlinear Oscil, 7, (2004), 318-325.

[45] D.R. Will and C.T.H. Baker, Stepsize control and continuity consistency for statedependent delay-differential equations, J. Comput. Appl. Math, 53, (1994), 163-170.

[46] J. Wu, Theory and Applications of Partial Functional Differential Equations, Springer, New York, 1996.

Mouffak Benchohra

Laboratory of Mathematics

Djillali Liabes University of Sidi Bel Abbes

Sidi Bel Abbes

Algeria

E-mail: benchohra@univ-sba.dz

Amel Heris

Laboratory of Mathematics

Djillali Liabes University of Sidi Bel Abbes

Sidi Bel Abbes

Algeria

E-mail: herisamel@gmail.com

Received: 24.10.2016

Accepted: 23.12.2016 